\title{
ON THE NUMBERS OF CUT-VERTICES AND END-BLOCKS IN 4-REGULAR GRAPHS
}

\author{
DingGuo WANG ${ }^{2,3}$ And Erfang Shan ${ }^{1,2}$ \\ ${ }^{1}$ School of Management, Shanghai University, Shanghai 200444, China \\ ${ }^{2}$ Department of Mathematics, Shanghai University, Shanghai 200444, China \\ ${ }^{3}$ College of Mathematics Science, Chongqing Normal University \\ Chongqing 400047, China \\ e-mail: efshan@shu.edu.cn
}

\begin{abstract}
A cut-vertex in a graph $G$ is a vertex whose removal increases the number of connected components of $G$. An end-block of $G$ is a block with a single cut-vertex. In this paper we establish upper bounds on the numbers of end-blocks and cut-vertices in a 4-regular graph $G$ and claw-free 4-regular graphs. We characterize the extremal graphs achieving these bounds.
\end{abstract}

Keywords: 4-regular graph, claw-free, cut-vertices, end-blocks.

2010 Mathematics Subject Classification: 05C35.

\section{REFERENCES}

[1] M.O. Albertson and D.M. Berman, The number of cut-vertices in a graph of given minimum degree, Discrete Math. 89 (1991) 97-100. doi:10.1016/0012-365X(91)90402-N

[2] B. Bollobás, Modern Graph Theory (New York, Springer-Verlag, 2001).

[3] L.H. Clark and R.C. Entringer, The number of cut vertices in graphs with given minimum degree, Discrete Math. 18 (1990) 137-145. doi:10.1016/0012-365X(90)90145-8

[4] K. Nirmala and A.R. Rao, The number of cut vertices in a regular graph, Cahiers Centre Etudes Reserche Oper. 17 (1975) 295-299.

[5] N. Achuthan and A.R. Rao, On the number of cut edges in a regular graph, Australas. J. Combin. 27 (2003) 5-12.

[6] A. Ramachandra Rao, An extremal problem in graph theory, Israel J. Math. 6 (1968) 261-266. doi:10.1007/BF02760258 
[7] A. Ramachandra Rao, Some extremal problems and characterizations in the theory of graphs, Ph.D. Thesis, Indian Statistical Institute (1969).

[8] S.B. Rao, Contributions to the theory of directed and undirected graphs, Ph.D. Thesis, Indian Statistical Institute (1970).

[9] O. Suil and D.B. West, Balloons, cut-edges, matchings, and total domination in regular graphs of odd degree, J. Graph Theory 64 (2010) 116-131.

doi:10.1002/jgt.20443

Received 21 May 2012

Revised 8 January 2013

Accepted 8 January 2013 